Probing photo-ionization: experiments on positive streamers in pure gases and mixtures

This article has been downloaded from IOPscience. Please scroll down to see the full text article.

2010 J. Phys. D: Appl. Phys. 43145204

(http://iopscience.iop.org/0022-3727/43/14/145204)

The Table of Contents and more related content is available

Download details:

IP Address: 192.16.184.152

The article was downloaded on 24/03/2010 at 14:50

Please note that terms and conditions apply. 


\title{
Probing photo-ionization: experiments on positive streamers in pure gases and mixtures
}

\author{
S Nijdam ${ }^{1}$, F M J H van de Wetering ${ }^{1}$, R Blanc ${ }^{1,2}$, E M van Veldhuizen ${ }^{1}$ \\ and $\mathbf{U}$ Ebert $^{1,3}$ \\ ${ }^{1}$ Eindhoven University of Technology, Department Applied Physics, PO Box 513, 5600 MB Eindhoven, \\ The Netherlands \\ ${ }^{2}$ Ecole Polytechnique de l'Université d'Orléans, Orléans, France \\ ${ }^{3}$ Centrum Wiskunde and Informatica, Amsterdam, The Netherlands \\ E-mail: s.nijdam@tue.nl
}

Received 4 December 2009, in final form 8 February 2010

Published 23 March 2010

Online at stacks.iop.org/JPhysD/43/145204

\begin{abstract}
Positive streamers are thought to propagate by photo-ionization; the parameters of photo-ionization depend on the nitrogen : oxygen ratio. Therefore we study streamers in nitrogen with $20 \%, 0.2 \%$ and $0.01 \%$ oxygen and in pure nitrogen as well as in pure oxygen and argon. Our new experimental set-up guarantees contamination of the pure gases to be well below 1 ppm. Streamers in oxygen are difficult to measure as they emit considerably less light in the sensitivity range of our fast ICCD camera than the other gases. Streamers in pure nitrogen and in all nitrogen-oxygen mixtures look generally similar, but become somewhat thinner and branch more with decreasing oxygen content. In pure nitrogen the streamers can branch so much that they resemble feathers. This feature is even more pronounced in pure argon, with approximately $10^{2}$ hair tips $\mathrm{cm}^{-3}$ in the feathers at $200 \mathrm{mbar}$; this density can be interpreted as the free electron density creating avalanches towards the streamer stem. It is remarkable that the streamer velocity is essentially the same for similar voltage and pressure in all nitrogen-oxygen mixtures as well as in pure nitrogen, while the oxygen concentration and therefore the photo-ionization lengths vary by more than five orders of magnitude. Streamers in argon have essentially the same velocity as well. The physical similarity of streamers at different pressures is confirmed in all gases; the minimal diameters are smaller than in earlier measurements.
\end{abstract}

S Online supplementary data available from stacks.iop.org/JPhysD/43/145204/mmedia

(Some figures in this article are in colour only in the electronic version)

\section{Introduction}

\subsection{Positive streamers and photo-ionization}

Streamers are the first stage of electric breakdown, when a high voltage is applied to gas volumes [1-3]. The discharge can later develop into spark or lightning, but it also can stay completely in the streamer phase. An example of a discharge that remains essentially in the streamer phase is a sprite discharge, a huge discharge at 40 to $90 \mathrm{~km}$ altitude above thunderclouds $[4,5]$. In a wide field of technical applications, the voltage pulse is intentionally kept short to suppress inefficient gas heating during later stages of the discharge [6,7], and this technology builds on streamers. Streamers enhance the electric field at their tip to values above the breakdown value and create a region of active local ionization dynamics; in nanosecond resolved intensified CCD-photographs, these active areas can be seen as bright spots [8-10]. For further reading on streamers and sprites, we refer to a recent cluster issue in J. Phys. D: Appl. Phys. [11] and to the AGU Chapman conference on Effects of Thunderstorms and Lightning in the Upper Atmosphere (Pennsylvania, May 2009) [12] with its 
forthcoming issue in J. Geophys. Res. as well as to the many original papers cited there.

While negative streamers naturally propagate through electron drift (possibly supported by additional mechanisms), positive streamers are typically easier to generate, but more difficult to explain [13], as they propagate against the electron drift direction with velocities comparable to this velocity. While Townsend [14] in 1915 still assumed symmetry between positive and negative charge carriers, it soon became clear that positive ions were not suitable for impact ionization and too slow. The commonly accepted explanation for the propagation of positive streamers in air is photo-ionization; this was suggested in 1935 by Flegler and Raether in Munich [15], by Bradley and Snoddy in Virginia [16] and by Cravath in California [17]: the active ionization region emits UV radiation that at some distance (in particular, ahead of the front) can generate additional electron-ion pairs. The theoretical understanding of photo-ionization in nitrogenoxygen mixtures was quantified by Teich $[18,19]$ : the energetic electrons in the high field region of the streamer excite certain levels of molecular nitrogen with energies above the ionization energy of oxygen. The levels deexcite by emission of a photon that can ionize molecular oxygen. Teich determined the dominant wave lengths, and he also identified quenching at higher air pressures as a mechanism suppressing photoionization.

There has hardly been any experimental work published on measuring photo-ionization directly in the past 40 years. Cravath [17,20] already in 1935 suggested two ionization lengths of 1 and of $5 \mathrm{~mm}$ in air at standard temperature and pressure, and Raether [21] measured $5 \mathrm{~mm}$ in 1938 where he also investigated hydrogen and oxygen. After the second world war, Przybylski and Teich continued this work in Raether's lab in Hamburg and published their results in German in 1958 and 1967 [18, 19, 22]. Penney and Hummert [23] in Pennsylvania investigated the process again and found in 1970 full agreement with the earlier measurements of Przybylski and Teich, and with those of Sroka in 'pure' oxygen, 'pure' nitrogen and air. The results show that photo-ionization in air is about 1 or 2 orders of magnitude more effective than in pure oxygen or pure nitrogen, but they still see significant photo-ionization in both pure gases. However, the only information about the purity of the gases is that 'commercial-grade' gases have been used. This probably refers to purities in the order of $0.1 \%$ to $1 \%$. They do not discuss which mechanisms could be responsible for the photo-ionization in any of the gases, in contrast to Teich. In recent years Aints et al [24] have used the same method as Penney and Hummert to investigate the effects of water content in air on photo-ionization. Their results are similar to the results of Penney and Hummert, with small corrections for the effects of water content.

The data of Przybylski, Teich, Penney and Hummert and the theoretical understanding of Teich were merged by Zhelezniak et al [25] into a model of photo-ionization that nowadays is used in most streamer simulations [26-31].

On the other hand, the reliability of the photo-ionization data and of the resulting Zhelezniak model has been questioned; for a recent discussion, we refer to the introduction of Nudnova and Starikovskii [32]. Background ionization has been suggested as an alternative to photo-ionization; in early simulations, photo-ionization was even replaced by background ionization to reduce the computational complexity [33]. The background ionization could either be due to radioactivity and cosmic radiation, or due to left over charges at high repetition rates of the discharge as elaborated by Pancheshnyi [34]. In the present experiments, high repetition rates are avoided, we use a $1 \mathrm{~Hz}$ repetition frequency for all experiments.

As photo-ionization is a vital part of streamer theory, and as direct measurements are difficult, Luque et al [31] have suggested indirect measurements through studying the interaction of two streamer heads propagating next to each other: they typically would repel each other electrostatically, but could merge through the non-local effect of photoionization. In an attempt to confirm this theory, Nijdam et al have performed experiments with two streamers emitted from adjacent needles [35], but a common parameter regime has not yet been explored for theory and experiment, and the photo-ionization could not yet be investigated along these lines. Kashiwagi and Itoh have shown that UV and VUV radiation from a surface streamer discharge can trigger a synchronous streamer discharge [36]. They have found that radiation around $115 \mathrm{~nm}$ is most effective, which is somewhat higher than the limit of $102.5 \mathrm{~nm}$ that is associated with nitrogen-oxygen photo-ionization. However, in the case of surface discharges, photo-electron emission from the insulator surface itself can replace photo-ionization of oxygen molecules in bulk streamer discharges. The photo-electron emission from the surface can occur at longer wavelengths than the bulk photo-ionization of oxygen molecules.

Here we follow a different experimental track. According to Teich's photo-ionization mechanism in nitrogen-oxygen mixtures, the density of emitted photons is proportional to the nitrogen concentration and the absorption lengths of the photons are inversely proportional to the oxygen concentration. If positive streamers in air indeed propagate through this mechanism, one would expect that their properties change when the ratio of nitrogen and oxygen is changed. The main purpose of this paper is therefore to investigate positive streamers in varying nitrogen-oxygen mixtures experimentally. To set the limits, we also investigate streamers in pure nitrogen and in pure oxygen, and for comparison, we also investigate them in pure argon.

\subsection{Previous experiments on streamers in different gases}

Streamers in nitrogen-oxygen mixtures. Streamers in varying nitrogen-oxygen mixtures have been investigated before by Yi and Williams [37], Ono and Oda [38] and Briels et al [39, 40].

Yi and Williams [37] use a $130 \mathrm{~mm}$ plane-plane geometry with protruding point, where the protruding point is a $3.2 \mathrm{~mm}$ diameter rod ground to a sharp tip (radius $\approx 100 \mu \mathrm{m}$ ). They use both positive and negative voltages pulses with amplitudes between 70 and $130 \mathrm{kV}$ and rise times of the order of $50 \mathrm{~ns}$. Nitrogen-oxygen mixtures with oxygen fractions between ' $0 \%$ ' and $15 \%$ are investigated. The purity of their pure 
nitrogen is not specified but it is presumably significantly below $0.1 \%$, as this is the oxygen content of their next gas mixture. They claim that their measurements strongly suggest that photo-ionization plays an important role because (especially positive) streamers propagate faster at higher oxygen concentrations. For high voltages $(>100 \mathrm{kV})$, the propagation velocity of positive streamers increases with roughly a factor of five when going from their pure nitrogen to $10 \%$ oxygen in nitrogen. In negative streamers the same concentration change leads to a velocity increase of less than $40 \%$.

Ono and Oda [38] also use a point-plane geometry, but with a $13 \mathrm{~mm}$ gap. Their tips are made by cutting a $0.3 \mathrm{~mm}$ stainless steel wire and have no well-defined tip profile. They apply positive voltage pulses with amplitudes between 13 and $37 \mathrm{kV}$, rise times in the order of tens of nanoseconds and durations of a few hundred nanoseconds on nitrogen-oxygen mixtures containing oxygen fractions between ' $0 \%$ ' and $20 \%$. Again, the purity of the nitrogen used in the experiments is not specified. Their next purest mixture contains $0.2 \%$ oxygen. They claim that propagation velocity, diameter and shape of the streamers are strongly influenced by the oxygen concentration. No measured value varied by more than a factor of five when changing the oxygen concentration from $20 \%$ to $0 \%$. The streamer diameter increases from $0.2-0.4 \mathrm{~mm}$ in pure nitrogen to more than $1 \mathrm{~mm}$ in air. At $18 \mathrm{kV}$, the propagation velocity increases from $\sim 2 \times 10^{5} \mathrm{~m} \mathrm{~s}^{-1}$ in pure nitrogen to $5 \times 10^{5} \mathrm{~m} \mathrm{~s}^{-1}$ in air.

Briels et al $[39,40]$ have measured in air, a mixture with $0.2 \%$ oxygen in nitrogen and 'pure nitrogen'. They use 5 to $45 \mathrm{kV}$ positive pulses with rise times of the order of 20-200 ns in point-plane gaps of 40 and $160 \mathrm{~mm}$ at pressures between 100 mbar and 1 bar. They have shown that at lower-oxygen concentrations, streamers branch and zigzag more, they are brighter and thinner (about 40\% thinner in pure nitrogen than in air). They have not found a clear effect of oxygen concentration on propagation velocity; at 1 bar they are lowest in pure nitrogen, while at lower pressures they are the same or highest in pure nitrogen. However, the set-up that was used in these experiments is not designed for high purity gas handling. Therefore, it is not known what the exact purity of the pure nitrogen was in all of the experiments described by Briels et al.

In order to better investigate the effects of low oxygen concentrations, here we will present results in which the purity of the gases can be guaranteed to ppm (parts per million) levels.

Streamers in argon. Aleksandrov et al [41] have performed experiments on streamers in pure argon (99.99\% purity) and a mixture of 1 to $5 \%$ oxygen in argon, all at atmospheric pressure. They present streak photography of discharges in a $250 \mathrm{~mm}$ rod-plane gap with 10 to $60 \mathrm{kV}$ voltage pulse with $1 \mu$ s rise time. They conclude that a non-thermal mechanism of streamer breakdown in the case of argon discharges gives way to leader breakdown when $1 \%$ or more of oxygen is added. This addition also leads to a decay of the streamer channel that is an order of magnitude faster and therefore a noticeably higher electric field is needed for the streamers to bridge the gap. Their simulations show that this can be explained by the quenching of excited argon molecules by oxygen molecules. Van Veldhuizen and Rutgers [42] have used air and argon. They found that streamers in argon branch less and can only be produced in a narrow voltage range.

Measurements similar to the ones presented in this paper on two planetary gas mixtures (Venus and Jupiter) are presented in a paper by Dubrovin et al [43].

\subsection{Advantages of short pulses}

Past measurements by Briels et al [39, 40,44,45] and others $[37,38,41]$ have been performed with voltage pulses with durations of many hundred nanoseconds up to many microseconds and with rise times of tens of nanoseconds or more. This approach has two disadvantages: firstly, the slow rise time means that the streamers may initiate at a significantly lower voltage than the reported maximum voltage. Therefore, they may not exhibit the properties (such as diameter and propagating velocity) that are representative for this voltage, but for a lower voltage. Secondly, a long pulse duration may lead to a streamer-to-spark transition. Because a spark is usually much brighter than a streamer, it may damage the photo-cathode of an ICCD camera, and is therefore avoided.

One way to overcome these two problems is to use a power supply based on a Blumlein pulser $[46,47]$. With such a circuit, it is possible to make pulses with a well-defined duration (depending on the cable length) and a fast rise time (order 1 to $10 \mathrm{~ns}$ ). Briels et al have investigated the effect of rise time on streamer properties. We extend this to lower rise times.

\subsection{Content of the paper}

In this paper we present experimental measurements on streamer discharges in different gas mixtures, with an emphasis on nitrogen-oxygen mixtures, focusing on photo-ionization. We will repeat some measurements by Briels et al [40], but with a better defined purity of the gases and better optics to measure streamer diameters. We also extend the measured range to nitrogen of $1 \mathrm{ppm}$ purity and to argon and oxygen of $10 \mathrm{ppm}$ purity and we discuss implications for the propagation mechanism. We measure at various pressures to further test streamer similarity laws.

\section{Experimental set-up}

We have built a set-up that is specifically designed to ensure the purity of the enclosed gases. For this reason, the set-up can be baked to reduce out-gassing, it contains no plastic parts, except for the o-ring seals and it stays closed all of the time. When not in use, the set-up is pumped down to a pressure of about $2 \times 10^{-7}$ mbar. The leak rate (including outgassing) under vacuum is about $8 \times 10^{-7} \mathrm{mbar} \mathrm{min}^{-1}$, which is considerably lower than the $5 \times 10^{-3} \mathrm{mbar} \mathrm{min}^{-1}$ reported by Yi and Williams [37]. We have used a helium leak tester to check for any leaks, but have not found any.

During use, the gas inside the set-up is flushed with such a flow rate that all gas is replaced every $25 \mathrm{~min}$. The 


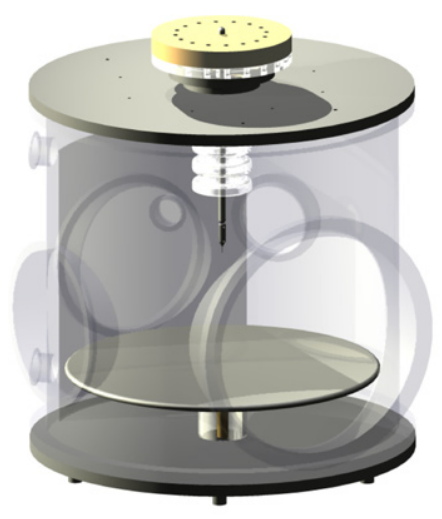

(a)

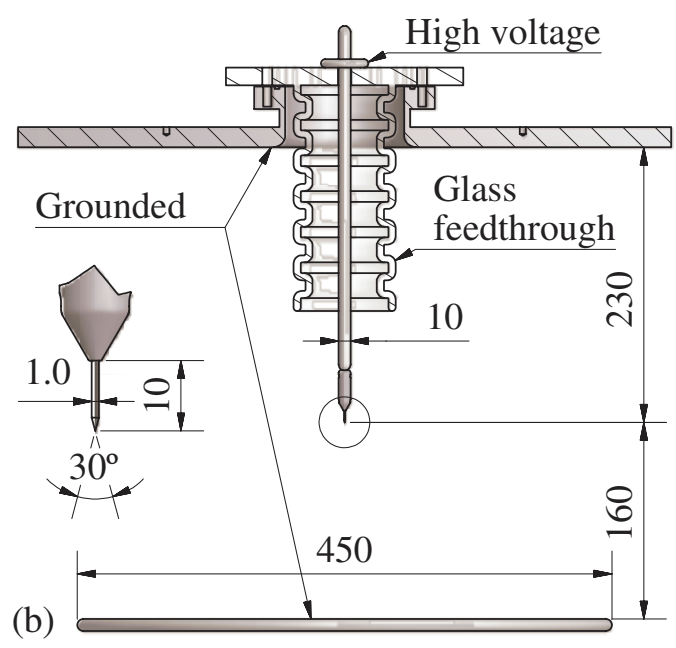

Figure 1. Overview ( $a$ ) and schematic view $(b)$ of the new high purity vacuum vessel with the ICCD camera. The wall of the vessel has been rendered transparent in the overview so that the anode tip and cathode plane are clearly visible. In the schematic view, dimensions are given in $\mathrm{mm}$. The bottom of the tip holder and the tungsten anode tip are enlarged on the left side of the image.

absolute flow rate is controlled by a mass-flow controller and depends on pressure. The flow rate is sufficiently high that the contamination caused by the leak/out-gassing rate is significantly below $1 \mathrm{ppm}$ for all pressures used. We use mixtures of $\mathrm{O}_{2}$ and $\mathrm{N}_{2}$ that are pre-mixed by the supplier. According to the specifications, the amount of contamination is below $1 \mathrm{ppm}$. The mixtures we have used contain nitrogen with $20 \%, 0.2 \%, 0.01 \%$ and $<0.0001 \%$ oxygen (volumetric fractions). We refer to the $20 \%$ oxygen mixture as artificial air. Besides these mixtures, we have also used pure oxygen and pure argon, both with not more then $10 \mathrm{ppm}$ impurity.

The vacuum vessel contains a sharp tungsten tip, placed $160 \mathrm{~mm}$ above a grounded plane. A drawing of the vacuum vessel with the camera is given in figure 1. During a measurement, a voltage pulse is applied to the anode tip. The vacuum vessel is located inside a Faraday cage in order to protect the camera and other sensitive electronics from the electromagnetic pulses of the pulse source.

A propagating streamer head emits light and the path of these heads can therefore be imaged onto a camera with a lens. Because the emitted light is often weak an intensified
CCD (ICCD) is needed. The intensifier also enables us to take images with very short (nanosecond) exposure times. These images only show a small section of the streamer propagation and can therefore be used to measure the velocity of the streamers [45].

In our case, streamer discharges are imaged by a Stanford Computer Optics 4QuikE ICCD camera. We use two different lens assemblies: a Nikkor UV $105 \mathrm{~mm} f / 4.5$ camera lens mounted directly on the camera and a $250 \mathrm{~mm}$ focal length, $50 \mathrm{~mm}$ diameter achromatic doublet on an optical rail. The latter is used to zoom in on a specific region of the discharge in order to measure small streamer diameters. We use a window of conducting indium tin oxide (ITO) glass as part of the Faraday cage to protect the camera against the high voltage pulses.

In the images presented here, the original brightness is indicated by the multiplication factor Mf, similar to what Ono and Oda introduced in [38]. This value is a measure for the gain of the complete system, it includes lens aperture, ICCD gain voltage and maximum pixel count used in the false-colour images. An image with a high $\mathrm{Mf}$ value is in reality much dimmer than an image with similar colouring, but with a lower Mf value (the real brightness is proportional to $\mathrm{Mf}^{-1}$ for an image with the same colours). We have normalized the Mf value in such a way that the brightest image presented in this paper has an Mf value of 1 .

\subsection{Pulse sources}

We have used two different electric circuits to produce a voltage pulse: the so-called C-supply and a newly designed circuit based on a Blumlein pulser with a multiple sparkgap.

The C-supply consists of a $1 \mathrm{nF}$ capacitor that is charged by a high voltage negative dc source. Now a trigger circuit triggers a spark gap, which acts as a fast switch. The capacitor is discharged and puts a positive voltage pulse on the anode tip. This pulse has a $10-90 \%$ rise time of at least $15 \mathrm{~ns}$ and a fall time of about $10 \mu \mathrm{s}$, depending on discharge impedance and choice of resistors. This circuit is treated extensively in [44].

The Blumlein pulser is a new design based on existing knowledge about such circuits $[46,47]$. It produces a more or less rectangular pulse with a short rise time and a fixed duration. The present design allows for a rise time of about $10 \mathrm{~ns}$ and a pulse duration of $130 \mathrm{~ns}$, see figure 3 . This is achieved by charging two coaxial cables (see figure 2) with a dc voltage source. When the multiple sparkgap switch [48] at the end of one of these cables is closed, a voltage pulse will travel along the cable mantles. This voltage pulse will arrive at the anode tip. After traversing the second cable back and forth, the voltage pulse at the anode tip will be nullified. We see only one reflected pulse occurring after the first pulse (starting at $t \approx 250 \mathrm{~ns}$ ). This indicates that our system is quite well matched.

This new design has three advantages compared with the C-supply: the faster rise time ensures that the voltage during initiation and early propagation is closer to the reported maximum voltage; therefore thicker streamers are generated [44]. The short duration of the pulse enables us to 


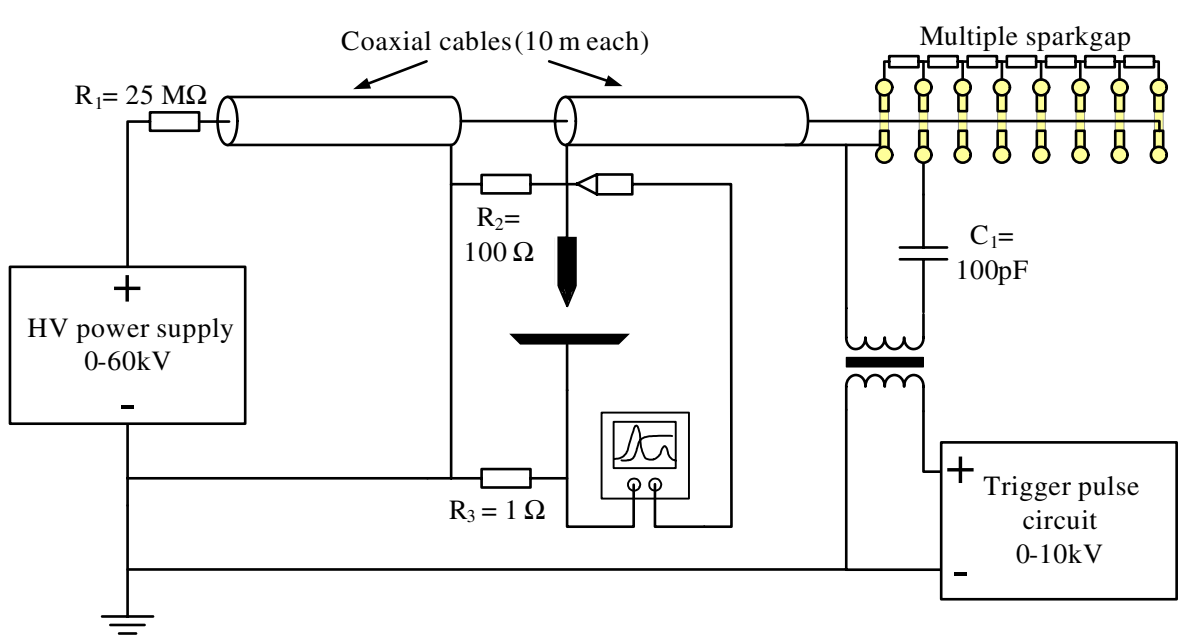

Figure 2. Schematic circuit of the Blumlein pulser design. The trigger pulse circuit has been omitted for sake of simplicity.

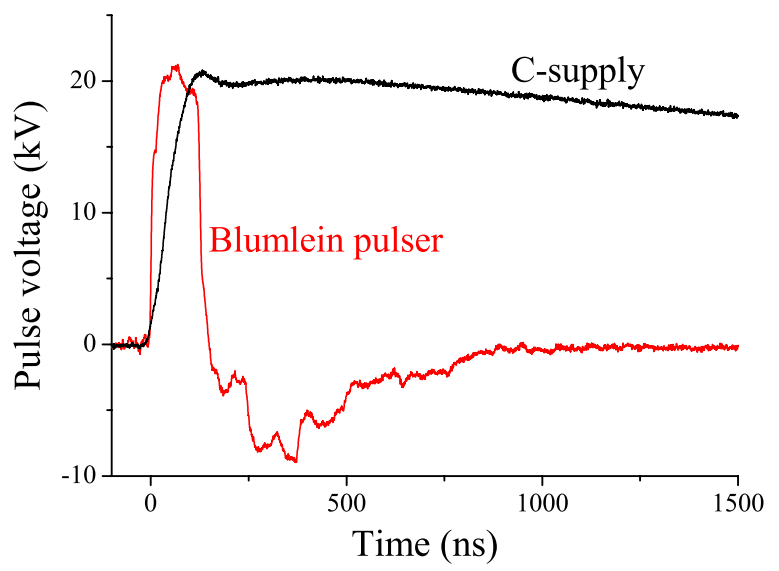

Figure 3. Comparison of the voltage pulses produced by the two different pulse sources. Note that the duration of the pulse from the C-supply depends largely on the impedance of the discharge and can therefore vary significantly according to gas type and pressure. Because the Blumlein pulser contains a low-ohmic resistor in parallel to the discharge, it is hardly influenced by the discharge impedance.

use overvolted gaps without the risk of spark formation (this can be dangerous for the camera). The short duration also helps to ensure that only primary streamers are produced. This makes it much easier to perform spectroscopic measurements on only these primary streamers; we can use long integration times without capturing other phenomena such as secondary streamers or glow discharges. However, this last advantage is only valid if the streamers do not bridge the gap long before the end of the pulse duration. In our measurements this is not always the case, as is shown in figure 9. The length of the pulse can be varied by using other lengths for the two coaxial cables, although this takes some time and is not easy to implement during a measurement series.

\subsection{Measuring streamer diameter and velocity}

From the images taken, the streamer diameter is determined with the following method:

- A straight streamer channel section is selected.
- In this section, several perpendicular cross sections of the streamer are taken.

- These cross sections are averaged so that they form one single cross section.

- The streamer diameter is determined as the full width at half maximum (FWHM) of the peak (streamer intensity).

A diameter of at least 10 pixels in the image is required to be sure that the streamer diameter is measured correctly and camera artefacts are negligible. For higher pressures (with thinner streamers), we have used the doublet lens in order to zoom in on a small specific region of the discharge.

The streamer propagation velocity has been measured with two different methods. The first method is as follows: we take short exposure images of streamers while they propagate in the middle of the gap (see figure 4). We then choose the thinner, straighter and longer streamer sections in each picture. The thinner streamers are ensured to be in focus, the longer streamers are assumed to propagate almost in the photograph plane. The length of each such streamer section is measured (we use a similar FWHM method for the diameter measurement), and the velocity is calculated as the ratio between length and exposure time, corrected for head size, depending on exposure time. Streamer sections that contain a branching event are ignored.

This method could be improved by using stereophotographic techniques as demonstrated in [35, 49]. However, for the sake of simplicity, we have chosen not to do this in the measurements presented here.

In the case of measurements on streamer velocity in argon, this first method does not work because of the long lifetimes of the excited states of argon. Even with a very short integration time and a delay long enough to start observing when the streamers are halfway through the gap, one will still see the entire trail between the streamer head and the anode tip. Therefore we have to measure the velocity by measuring the distance travelled by the fastest streamer heads as a function of time. When we now differentiate this curve we have the velocity as a function of time. For this method to work we need to have little jitter in the streamer initiation. This method 


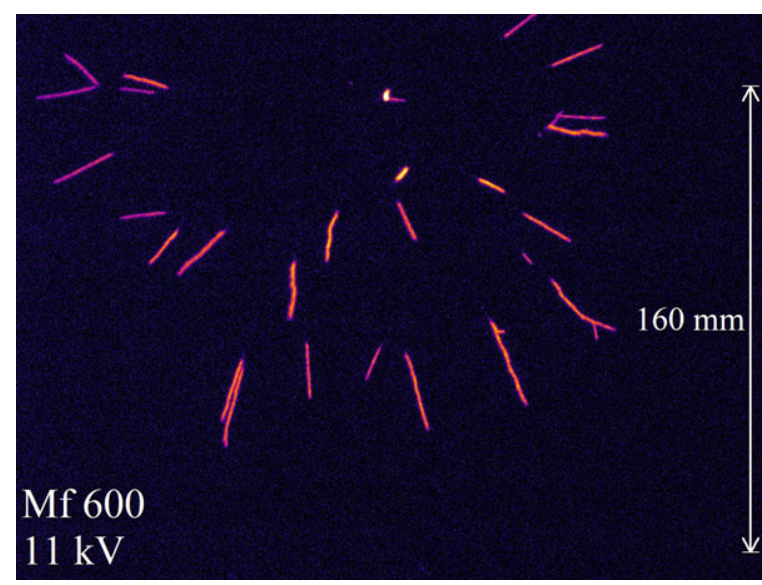

Figure 4. Example of an ICCD image used to determine streamer propagation velocities in nitrogen-oxygen mixtures. The velocity is calculated by dividing the length of the longer streamer sections by the exposure time. This example shows an image of the $0.2 \%$ oxygen in nitrogen mixture at 200 mbar with an exposure time of 300 ns.

has previously been used by Winands et al $[45,50]$ to measure streamer propagation velocities.

With both methods one should keep in mind that streamer velocity is not constant in the strongly non-uniform field of our point-plane discharge gap (see, e.g., figure 7 in [40] and accompanying discussion there). However, after roughly half of the gap, the velocity does not change much, except when the streamers get close (a few streamer diameters) to the cathode plate. Therefore, we have chosen to use images in which the streamers are roughly halfway into the gap (for both methods). We have verified that both methods described above give the same results for gases that support the first method.

More information about the discharge vessel, experiment timing, imaging system and measurement techniques can be found in $[35,40,45]$.

\section{Results and discussion}

\subsection{Images and general morphology}

We investigate the general morphology of the streamers by means of ICCD camera images. Previous time resolved photography in a similar set-up has established the following sequence of discharge evolution: first a glowing ball or initiation cloud appears at the needle electrode, the ball extends and transits into an expanding glowing shell that eventually becomes unstable and breaks up into many simultaneously propagating streamers $[40,51]$. The sizes of the initiation cloud and glowing shell scale inversely with pressure. At high pressures (1000 mbar in our case), they are so small that they cannot be distinguished in our images of the $6 \mathrm{~cm}$ gap. At low pressures, or when the size of the gap is very small, the initiation cloud and glowing shell may extend over more than half the gap. Pressure, gas mixture, gap length, voltage and voltage rise time determine whether a single, a few or many streamers emerge from the glowing shell.

In gas mixtures containing nitrogen, most radiation is produced by molecular nitrogen. It is well known that the streamer heads only emit light for a very short time (less than $2 \mathrm{~ns}$ ) in these mixtures $[45,52,53]$. We can assume that the atoms or molecules that emit the radiation have not moved significantly between excitation and emission. For example, the thermal velocity of a nitrogen molecule at room temperature is about $500 \mathrm{~m} \mathrm{~s}^{-1}$. This means that the maximum distance it can travel within $10 \mathrm{~ns}$ is $5 \mu \mathrm{m}$ if it is not scattered on its path; this is clearly below our resolution. Therefore, the image represents a mapping of the spatial distribution of the relevant excited states in nitrogen. Some time resolved pictures will be shown later in this paper. First we present time integrated ICCD pictures of such single discharge events.

As already discussed above, in argon the streamer channels remain bright tens to hundreds of nanoseconds after the streamer head has passed. However, even in this case the maximum distance travelled by an excited atom or molecule before it decays will still be smaller than the pixel size of our camera. Therefore, in all cases, the images represent a map of the locations where the excited molecules or atoms are created and the images are not influenced by diffusion of these excited species after excitation.

Nitrogen-oxygen mixtures and pure nitrogen. Figure 5 shows an overview of streamers made with the C-supply in the six different gas mixtures. The general morphology of the $\mathrm{N}_{2}: \mathrm{O}_{2}$ mixtures and pure nitrogen is very similar, although it is clear that, especially at low pressures, there are more branches in pure nitrogen and the $0.01 \%$ mixture than in the other two mixtures. This has also been observed by Ono and Oda [38]. Their streamer channels become marginally straighter for higher concentrations of oxygen.

One striking feature in the $\mathrm{N}_{2}: \mathrm{O}_{2}$ mixtures and the pure nitrogen is the maximal length of the streamers at 1000 mbar; the streamers are longest for the $0.2 \% \mathrm{O}_{2}$ mixture. However, the interpretation is not straightforward. In these images the exposure time of our camera was about $2 \mu \mathrm{s}$ and the decay time $(1 / e)$ of the voltage pulse was about $6 \mu \mathrm{s}$. On the other hand, Briels et al [40] in their 'pure nitrogen' have found much longer streamers under similar conditions that propagated for more than $4 \mu$ s after the start of the pulse. In those measurements the decay time of the voltage pulse was probably much longer than $6 \mu$ s because they used a higher parallel impedance in the C-supply circuit (the exact value is unclear). The lengths of our streamers in pure nitrogen can therefore be determined either by the exposure time of the camera or by the decay time of the voltage pulse. In air, streamers stay short both in Briels' and in the present measurements. This is probably due to the conductivity loss inside the streamer channel due to the attachment of electrons to oxygen molecules. In air, this loss mechanism is much stronger than in pure nitrogen with a small amount of oxygen contamination.

The images of the $0.2 \% \mathrm{O}_{2}$ and $0.01 \% \mathrm{O}_{2}$ are the brightest at 1000 mbar and (less so) at 200 mbar. This confirms the results of Ono and Oda [38]. One should be careful when comparing exact Mf values of our measurements. We have not conducted a statistical study into the brightness of the streamer channels. 

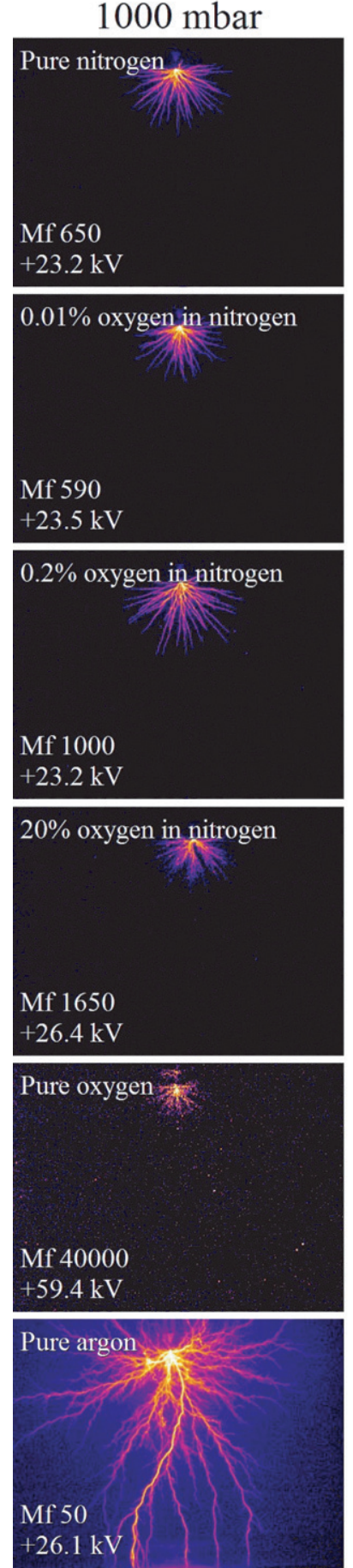
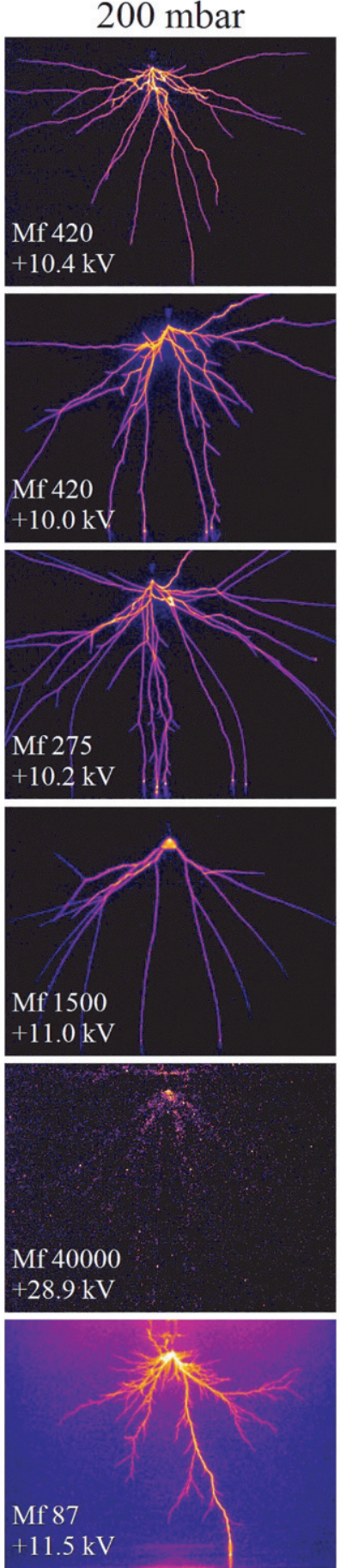
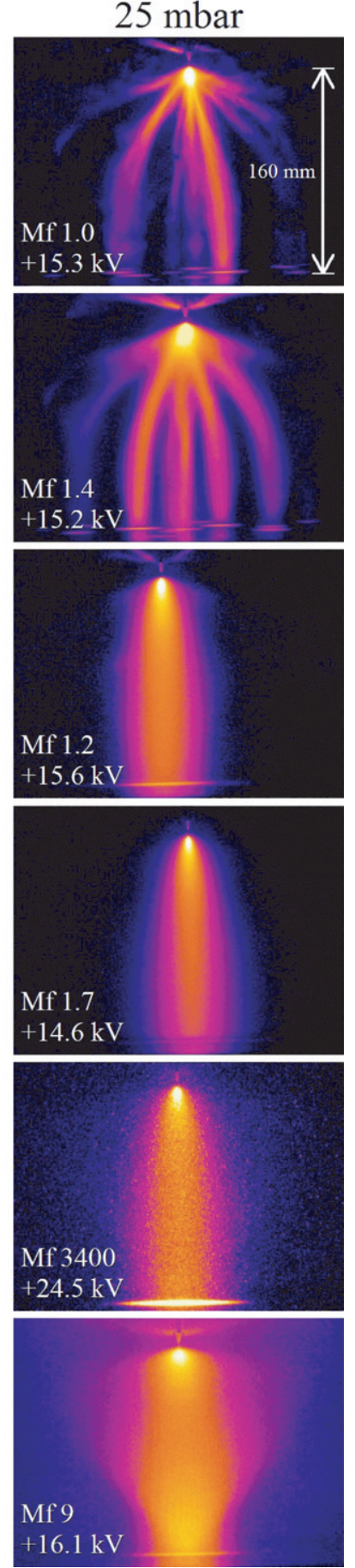

Figure 5. Overview of streamer discharges produced with the C-supply for all six gas mixtures used (rows), at 1000, 200 and 25 mbar (columns). All measurements have a long exposure time and therefore show one complete discharge event, including transition to glow for 25 mbar. The Mf gives an indication of the real intensity of the discharge. The white arrow at the top right image indicates the vertical position of the anode point and the cathode plane. Note that the voltages used are significantly higher for the oxygen measurements. At 25 mbar the discharge is partly in the glow phase instead of the streamer phase. Images at other voltages can be found in the supplementary data (stacks.iop.org/JPhysD/43/145204/mmedia).

Pure oxygen. The images of pure oxygen are clearly more noisy than all others because of the low light intensity of the streamers, which requires high multiplication factors. In order to make the images we had to increase the gain of the ICCD camera to close to its maximum level. The intensity of the streamers in pure oxygen is 2 to 3 orders lower than for the streamers in other gases. When the voltage of the pure oxygen measurement had been around $25 \mathrm{kV}$ as for the other gases, this difference would be even larger. However, at $25 \mathrm{kV}$ nothing could be seen and therefore we have used a $59.4 \mathrm{kV}$ image. At 200 mbar, the situation was similar.

Note that the measured intensity also depends on the emission spectrum, as a camera always has a wavelength dependent response. Our camera is sensitive between 200 and 


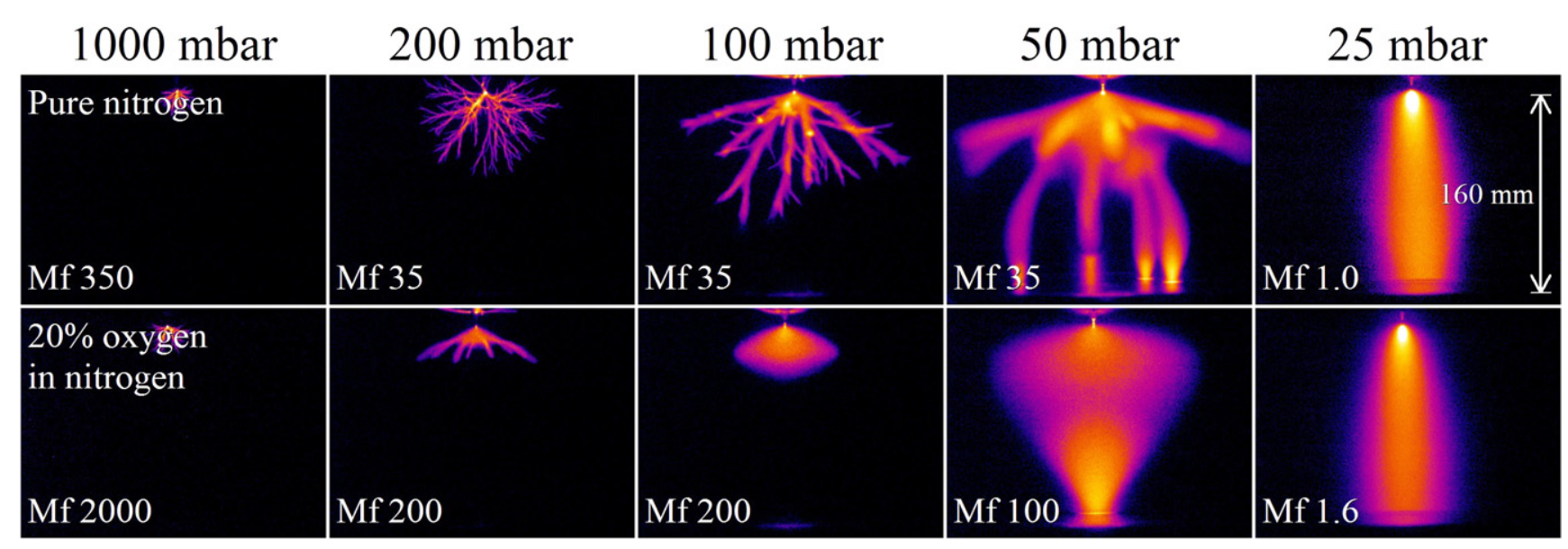

Figure 6. Overview of streamer discharges produced with a $20 \mathrm{kV}$ pulse by the Blumlein pulser ( $\approx 130 \mathrm{~ns}$ pulse length) for pure nitrogen (top row) and artificial air (bottom row), at five different pressures. All measurements have a long exposure time and therefore show the whole discharge.

$800 \mathrm{~nm}$. However, because of the ITO glass in our Faraday cage, radiation below $300 \mathrm{~nm}$ is cut off.

The low intensity of the streamers in oxygen can be explained by the lack of emission lines from oxygen in the sensitive region of the camera. The strongest line at $777 \mathrm{~nm}$ is close to the limit of the camera sensitivity curve. This makes it nearly impossible to do any quantitative research on streamers in pure oxygen with our camera. The images at pressures above $50 \mathrm{mbar}$ are not suitable for diameter or velocity measurements. At high pressures it is often even difficult to determine whether streamers are present or not. Therefore we will not present any other data on pure oxygen streamers.

Pure argon. Pure argon has a significant light emission intensity and yields good quality camera images. In comparison with the other gases, it branches much more, but most branches are very short. It also develops easily into a spark, which means that we have to take care that the voltage pulse remains short enough to avoid this (we did not use the Blumlein pulser with argon). As already discussed above, in argon the streamer channels remain bright tens to hundreds of nanoseconds after the streamer head has passed. However, even in this case the maximum distance travelled by an excited atom or molecule before it decays will still be smaller than the pixel size of our camera. Therefore, in all cases, the images represent a map of the locations where the excited molecules or atoms are created and the images are not influenced by diffusion of these excited species after excitation. In the 1000 and 200 mbar images we can already see that one channel is much brighter than the others. The relatively bright channel is the reason for the relatively low Mf values for argon at these pressures. This near-sparking behaviour is frequently observed in the argon discharges.

The easy sparking and heavy branching are probably due to the fact that argon is a noble gas without rotational or vibrational degrees of freedom, as we will discuss in more detail in section 4 . Therefore there are few inelastic collisions at low electron energies, and hence impact ionization becomes effective at much lower field strengths $E$ than in nitrogen or oxygen. The impact ionization coefficient $\alpha(E)$ is more than two orders of magnitude larger for argon than for nitrogen at fields below $30 \mathrm{kV} \mathrm{cm}^{-1}$ at atmospheric pressure [54].

Results from the Blumlein pulser. The differences between artificial air and pure nitrogen can be studied better with the Blumlein pulser than with the C-supply. We recall that the Blumlein pulser has a faster rise time and a shorter pulse duration than the $\mathrm{C}$-supply. This allows us to use the same voltage at all pressures without danger of provoking a spark. Hence in figure 6, the electric fields $E$ are the same up to modifications due to the presence of the discharge while the reduced fields $E / N$ increase with decreasing density $N$.

This figure shows that at 25 and 1000 mbar the images for pure nitrogen and artificial air are quite similar, but all intermediate pressures show clear differences between the two gases. Again, artificial air has fewer and thicker streamers, as well as a much larger initiation cloud or glowing shell (see discussion at the beginning of this section). Because of the limited duration of the pulse, no streamers emerge from the glowing shell in artificial air at 100 mbar. This limited duration is also the reason for the short streamer lengths at pressures above 50 mbar for both gases. We have used a voltage of $20 \mathrm{kV}$ in all measurements in figure 6; this voltage is higher than in figure 5 for all pressures below 1000 mbar.

The differences in appearance between air and pure nitrogen with the Blumlein pulser are also more pronounced than found by Briels et al [40]. This can again be attributed to the effect of a higher voltage and faster rise time in our case, but also to the higher purity that can be achieved in the new set-up. It seems that the thinnest or minimal streamers are similar in the different mixtures, but that air produces thicker streamers more easily.

The larger 'initiation cloud' in artificial air can also be observed in the 200 mbar images from figure 5 , when comparing this with the lower-oxygen concentration mixtures.

In figure 6 , in pure nitrogen at 25 mbar there is only one channel instead of the multiple channels in figure 5. This is caused by the higher voltage $(20 \mathrm{kV}$ versus $15.3 \mathrm{kV})$ and faster rise time $(\sim 10 \mathrm{~ns}$ versus $\sim 50 \mathrm{~ns})$ from the Blumlein pulser 

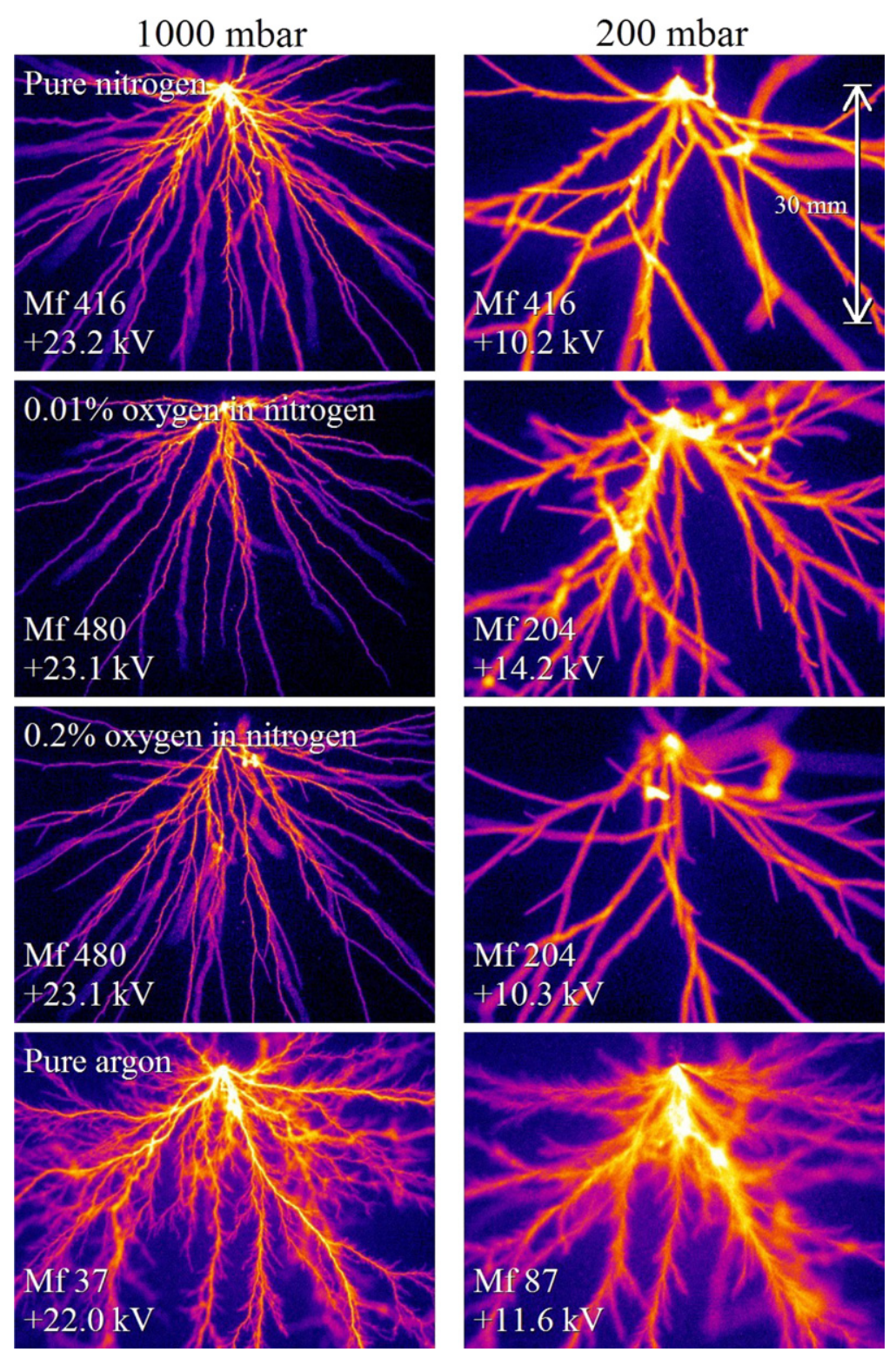

Figure 7. Zoomed images produced with the C-supply for four different gases (rows), at 1000 and $200 \mathrm{mbar}$ (columns) around the anode tip region (see length indication at top right image). All other settings are similar to figure 5 . Note that the image for $0.01 \%$ oxygen at $200 \mathrm{mbar}$ has a deviating voltage.

compared with the C-supply. Such a high voltage would lead to a spark when using the C-supply.

The shapes of all discharges at pressures of 50 mbar and lower correspond to the shapes found by Rep'ev and Repin [55] in discharges in a $100 \mathrm{~mm}$ point-plane gap in air at atmospheric pressure with $220 \mathrm{kV}$ pulses. So their reduced background field $(E / N)$ is quite similar to ours. They use a bullet-shaped electrode with a tip radius of $0.2 \mathrm{~mm}$.

Initiation delay and jitter. We have not observed any effects of gas mixture on initiation delay or jitter as reported by $\mathrm{Yi}$ and Williams [37]. In all cases, the streamer initiation jitter was limited to less than $10 \mathrm{~ns}$. This can probably be attributed to our electrode holder geometry, which induces more field enhancement at the tip, our fast voltage rise times and our overvolted gaps. The protrusion-plane electrode of Yi and Williams has a tip radius of about $100 \mu \mathrm{m}$ and protrudes only
$10 \mathrm{~mm}$ from a plane. Our tip has a radius of about $15 \mu \mathrm{m}$ and is mounted in an elongated holder (see figure 1). Van Veldhuizen and Rutgers [42] have shown that such a difference in geometry of the holder and tip can have a large influence on streamer initiation and propagation. Therefore, initiation was always fast in our case and we did not observe differences between the gas mixtures.

\subsection{Feather-like structures}

Figure 7 shows images from similar discharges as in figure 5 but now zoomed to the region closest to the anode tip. The zooming is achieved by moving the camera closer to the experiment. We omitted images from pure oxygen and from artificial air. The former is omitted because it is hardly visible, for the latter we unfortunately do not have any zoomed measurement images available at 200 mbar and voltages between 10 and $15 \mathrm{kV}$. We 

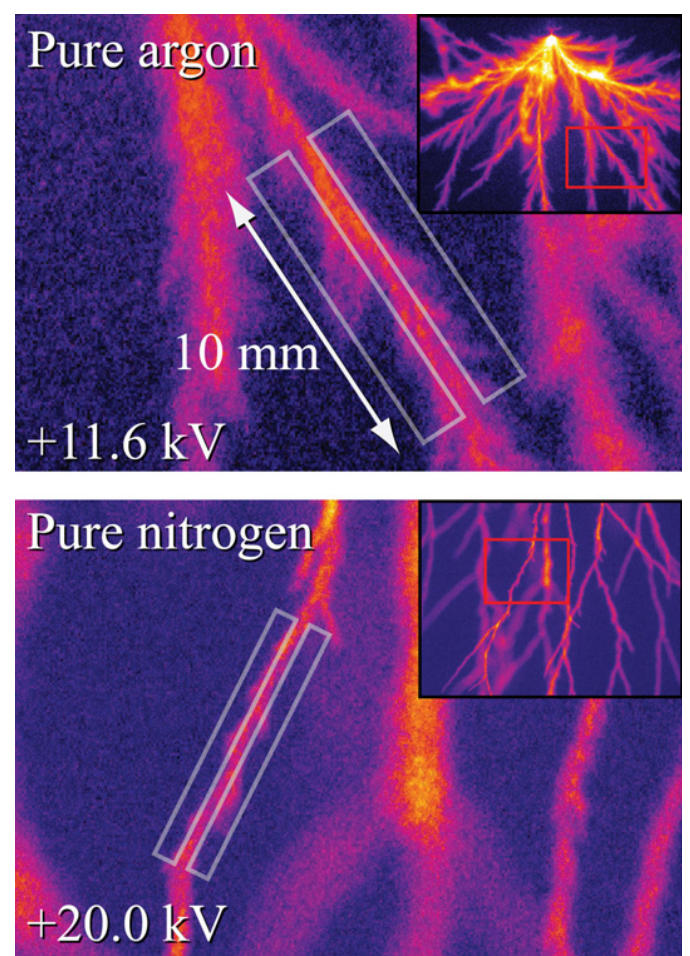

Figure 8. Feather like structures in a pure argon and a pure nitrogen discharge. Both images are acquired with the C-supply at 200 mbar. The images as captured by the camera are shown in the boxes in the top right corners with the zoomed areas indicated. The grey rectangles in the zoomed images indicate the area in which the number of small branches was counted. The nitrogen image is on the same scale as the argon image, but lower in the $160 \mathrm{~mm}$ gap (roughly halfway).

do have zoomed images of artificial air at 1000 mbar and $23 \mathrm{kV}$. These are very similar to the corresponding images of the $0.2 \%$ oxygen mixture. For 200 mbar, however, we do not expect that it is the same, as the unzoomed images from figure 5 already show large differences between these two gas mixtures, also close to the electrode tip region.

We observe again that for lower-oxygen concentrations, the streamers branch more often. In pure nitrogen at $200 \mathrm{mbar}$ they form shapes resembling feathers. In argon, these feather-like structures are even more pronounced, especially at 200 mbar. The fact that there are no straight, smooth streamer channels in the argon discharges makes it impossible to determine unambiguous streamer diameters in this gas.

We have analysed one channel from an argon image at 200 mbar (figure 8 ). We counted the number of side channels from a $10 \mathrm{~mm}$ long section of this channel in a region between 0.5 and $2 \mathrm{~mm}$ from the centre of the channel on both sides. Dividing this number by the volume of the counting region (we assume cylindrical symmetry of the streamer channel) leads to a branch density of about $10^{2} \mathrm{~cm}^{-3}$.

When we apply the same procedure to the image of a nitrogen discharge from figure 8 , we find roughly the same branch density. Although the number of small side channels is lower than in the argon image, so is their length. Therefore the counting region reaches only about $1 \mathrm{~mm}$ around the centre of the channel. We will discuss the interpretation of this phenomenon in section 4 .

\subsection{Time resolved images}

We took time resolved, movie-like (stacks.iop.org/JPhysD/43/ $145204 / \mathrm{mmedia}$ ) image sequences by decreasing the integration time of our camera and varying its internal delay value. Results of these measurements are given in figures 9 and 10 . All measurements were performed at about $20 \mathrm{kV}$ with the Blumlein pulser as described in section 2.1. Note that our camera can only take one image per discharge event and therefore these images are all from separate events. In figure 9, the streamer crosses the gap in less than $15 \mathrm{~ns}$. After this time, it evolves into a glow discharge, which is much brighter than the original streamer and initiation cloud. This glow discharge remains bright for the duration of the voltage pulse (130 ns), and then starts to extinguish.

After roughly $290 \mathrm{~ns}$, the discharge appears again. This is a negative discharge caused by the reflected pulse in the Blumlein pulser (see figure 3). Obviously, at this pressure, the gap is overvolted at $20 \mathrm{kV}$, which explains the high propagation velocity of about $3 \times 10^{6} \mathrm{~m} \mathrm{~s}^{-1}$.

At 100 mbar, the streamers do not reach the other side of the gap within the pulse duration. Therefore, the light is only emitted by the streamers and no glow discharge is formed, see figure 10 . The images at 102 and 150 ns clearly show that the light is only emitted by the propagating streamer heads, as was shown before by Briels et al [40].

\subsection{Effects of repetition frequency}

We have performed measurements on the effect of the pulse repetition frequency with frequencies down to $0.03 \mathrm{~Hz}$. An example of such a measurement can be seen in figure 11. In this measurement, the power supply was triggered with a repetition frequency of $1 \mathrm{~Hz}$. After a measurement, the triggering was stopped, and resumed $30 \mathrm{~s}$ later. Next, one image was stored, corresponding to either the trigger 1, 2 or 3 after the waiting period. We have not observed any influence of trigger number on streamer morphology and other properties (all images in one row of figure 11 are indistinguishable). This was tested and observed for pure nitrogen and for the $0.2 \%$ oxygen in nitrogen mixture. We have measured up until trigger 5, but this leads to the same results as triggers 1, 2 and 3 .

We have also seen that within a single series of subsequent voltage pulses, streamers follow a new (semi-random) route on every new voltage pulse. It seems that streamer channels do not have a tendency to follow the same path twice nor is there any indication that they are in any way influenced by the shape of the previous discharge (at $1 \mathrm{~Hz}$ repetition frequency). This is of course not the case for conditions that lead to only one streamer channel. In this case, the one channel is always located on the symmetry axis of the experimental setup.

Unfortunately we do not have any images to prove this statement, as, in our measurement procedure, images are stored manually and we have not stored any image sequences. However, during a measurement session, the images are shown on the screen with the repetition frequency of $1 \mathrm{~Hz}$ (this is then stopped to store an image). From our experience, consecutive images on the screen are completely independent from each other. Any tendency to follow old paths would be easily 

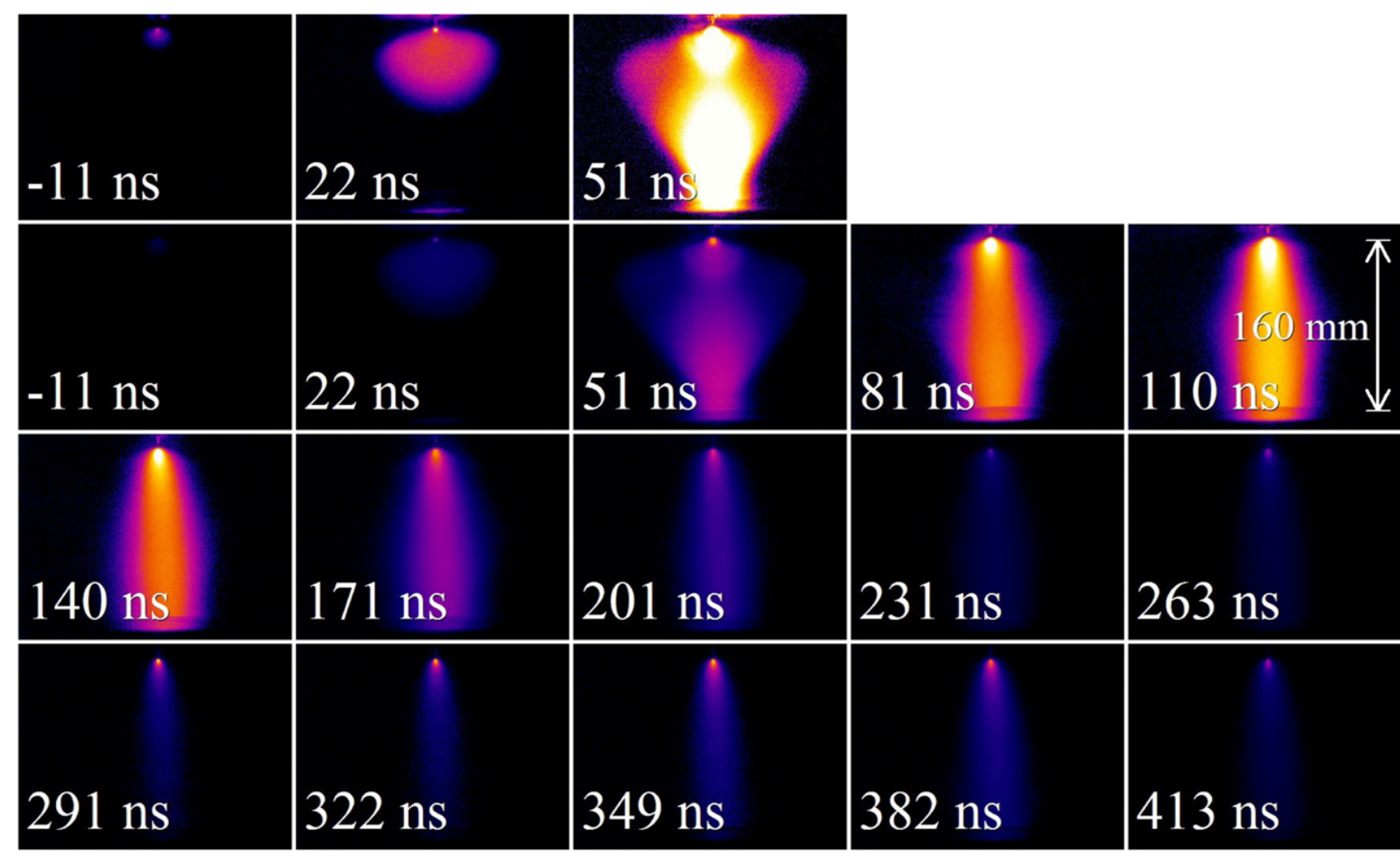

Figure 9. Image sequence of streamer discharges produced with a $20 \mathrm{kV}$ pulse by the Blumlein pulser in pure nitrogen at $25 \mathrm{mbar}$. The integration time of each image is $15 \mathrm{~ns}$ and the indicated time is the camera delay with respect to the beginning of the voltage pulse. The images in the top row are identical to the first three in the second row, except for their intensity representation. The images in the top row have an Mf value of 125, all other images have an Mf value of 8.6. An avi-movie of all recorded frames can be found in the supplementary data (stacks.iop.org/JPhysD/43/145204/mmedia).
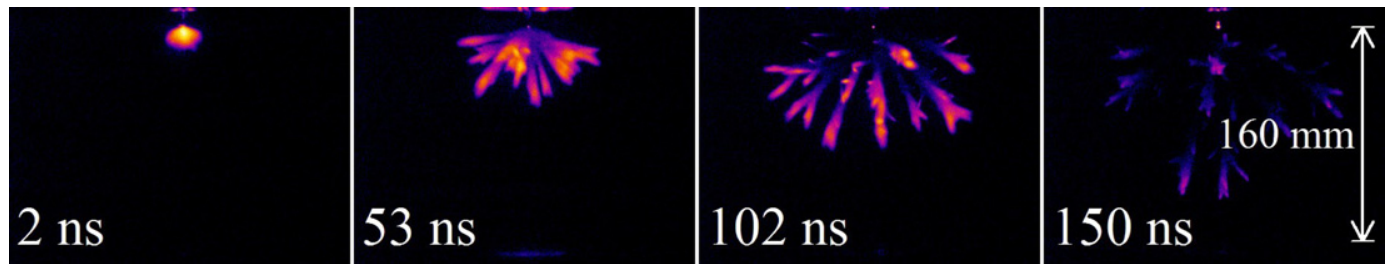

Figure 10. Image sequence of streamer discharges produced with a $20 \mathrm{kV}$ pulse by the Blumlein pulser in pure nitrogen at $100 \mathrm{mbar}$. All images have an Mf value of 158. Recall that we cannot capture more than one image per discharge event. Therefore the images presented here are all from separate discharges. An avi-movie of all recorded frames can be found in the supplementary data (stacks.iop.org/JPhysD/43/145204/mmedia).

observable, especially in situations with a limited number of streamer channels (e.g. 3-50). In more than six years of streamer measurements by at least seven different people, such behaviour has never been observed.

The only effect of repetition frequency that we have observed is that for certain conditions where streamer initiation is not easy because the applied voltage is close to the threshold, the chances of initiation are increased for repeated discharges. Under these conditions, the first image of a trigger series (trigger 1) is often completely empty or only contains a small glowing cloud around the electrode. Subsequent triggers do then often lead to a complete streamer discharge. It can be expected that the jitter (i.e. variations in initiation time) of the first few discharges in these conditions is also large, but we have not measured this. In most of our measuring conditions, the initiation jitter was below $10 \mathrm{~ns}$.
These effects regarding initiation may be attributed to leftover ionization or metastables from previous discharges. As the discharge always starts around the tip, any leftover ionization will have an influence on the initiation and maybe the early propagation of the streamer. However, we did not perform a detailed investigation of this phenomenon, as we are focusing on bulk streamer propagation and not initiation or other electrode effects.

\subsection{Minimal streamer width}

Briels et al have also shown that the reduced streamer diameter $n \cdot d_{\min }$ is constant as a function of the density $n$ for a specific gas mixture [40]. The choice for these thinnest channels has been made because experimental data as well as theoretical considerations show that there is a lower limit in streamer 
Trigger 1
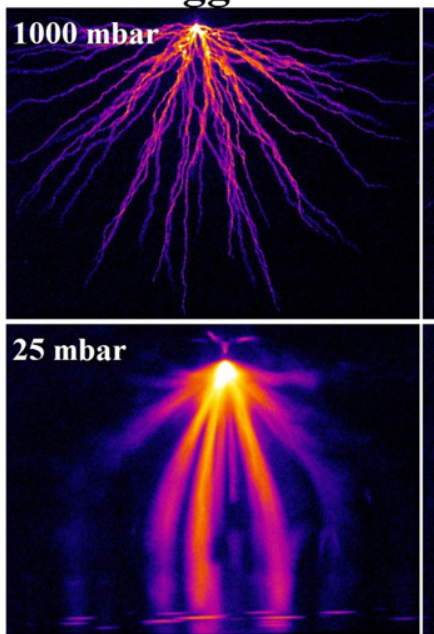

Trigger 2
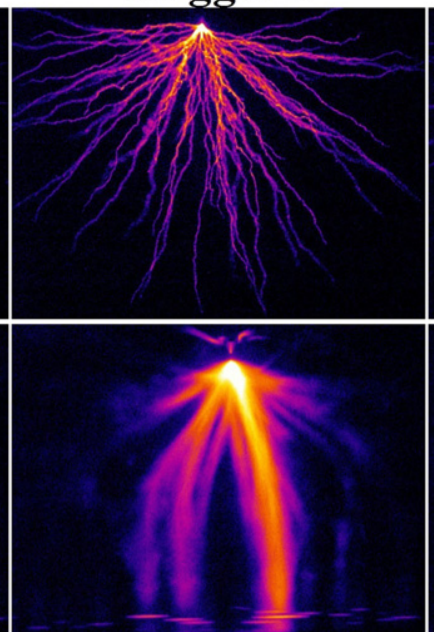

Trigger 3
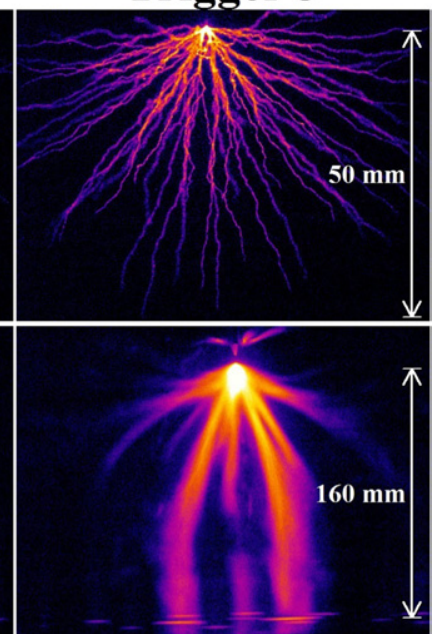

Figure 11. Discharge images as a function of trigger number. After a waiting period of about $30 \mathrm{~s}$, the discharge pulse generator is started with a repetition frequency of $1 \mathrm{~Hz}$. The images shown here are triggers 1, 2 and 3 after the waiting period. All images are taken in pure nitrogen with the C-supply. The images in the top row are zoomed images taken with $1000 \mathrm{mbar}_{2}, 40 \mathrm{kV}$ and have an $\mathrm{Mf}$ value of 350 . The images in the bottom row are full images taken with 25 mbar $\mathrm{N}_{2}, 15 \mathrm{kV}$ and have an Mf value of 1.4 . Note that the images in one row are not from the same series. We have only stored one image per measurement series.

diameter, but no upper limit of the streamer diameter with growing voltage has been found yet.

The minimal streamer diameters have been measured on images where the streamer diameter (FWHM) is at least 10 pixels wide. We took zoomed images like the $200 \mathrm{mbar}$ images from figure 7 for this purpose, by moving the camera closer to the discharge, and even higher magnifications at higher pressures. As stated before, we were unable to reliably measure diameters for streamers in pure oxygen and pure argon. Measurements of the minimal streamer diameters are given in figure 12. All results have been obtained with the C-supply. Some observations:

- The reduced diameter is roughly constant for any gas mixture, but it does increase slightly at higher pressures.

- The reduced diameter increases as a function of oxygen concentration although there is no significant difference between pure nitrogen and nitrogen with $0.01 \%$ oxygen.

The increase in reduced diameter as a function of pressure could be a measurement artefact: it is possible that the width of the streamers at high pressures is still overestimated because of bad focusing (shallow depth of field) and other imaging artefacts [44].

Ono and Oda [38] also find an increase in diameter although they report an increase of a factor of 12 between pure nitrogen and a $20 \%$ oxygen in nitrogen mixture. This large factor can be attributed to the fact that Ono and Oda do not specifically look for minimal streamers. They use a pulse of at least $15 \mathrm{kV}$ in gas at atmospheric pressure in a $13 \mathrm{~mm}$ point-plane gap. This is an overvolted gap, which will lead to streamers that are thicker than the minimal streamers we look at. We see as well that air streamers can be thicker than pure nitrogen streamers, but our minimal streamers are similar for all mixtures.

When we compare the values from figure 12 with the results of Briels et al [40] we see that they are lower in all cases.

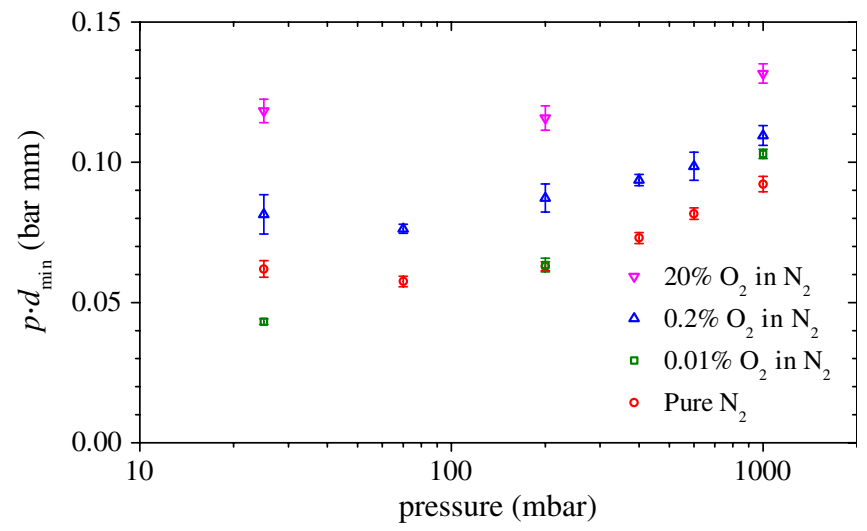

Figure 12. Scaling of the reduced diameter $\left(p \cdot d_{\min }\right)$ with pressure $(p)$ for the four different nitrogen oxygen mixtures. Every point represents 4 to 10 measured streamer channels each from a separate discharge image. The error bars give the standard error and do not include systematic errors. All measurements are performed at room temperature; therefore we use pressure instead of density to scale streamers.

Briels et al give an average value of $p \cdot d_{\text {min }}$ of 0.12 bar mm for 'pure' nitrogen and 0.20 bar $\mathrm{mm}$ for air. We have found 0.07 bar $\mathrm{mm}$ and 0.12 bar $\mathrm{mm}$, respectively. There are several reasons for the differences:

- Influences of other gas components such as water vapour and carbon dioxide that are more prevalent in the set-up of Briels than in the new high purity set-up.

- Small differences in voltage pulse rise time and amplitude that could lead to larger than minimal streamers in the case of Briels.

- Overestimation of streamer diameters by Briels because of the optical problems explained above.

Note that the pure nitrogen in the work of Briels et al was probably less pure than in this work. The new set-up is much 


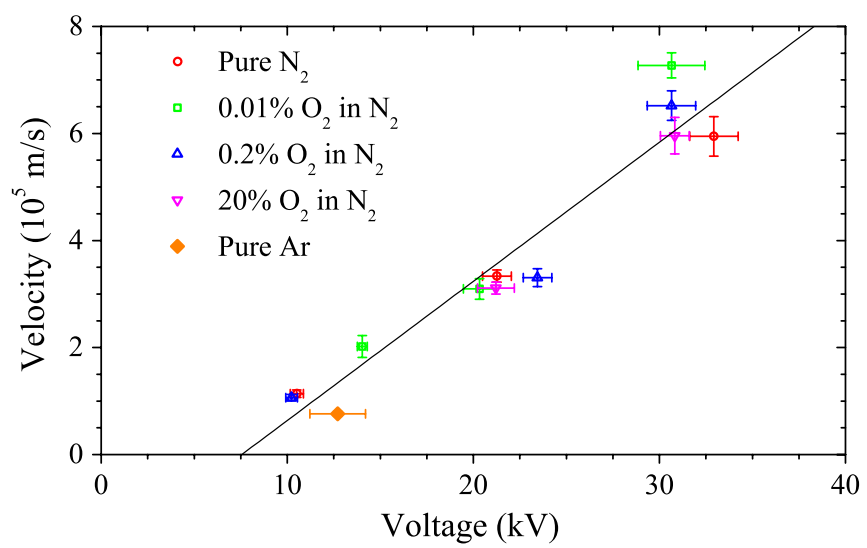

Figure 13. Velocity measurement results at $200 \mathrm{mbar}$ for five different gases. The horizontal error bars indicate the spread in the applied voltages, the vertical error bars indicate the sample standard deviation for 4-6 measurements. The velocities are measured roughly in the middle of the gap, except for the cases where the discharge does not reach this far (at lower voltages), in these cases, the velocity is determined at a position close to the end of the streamer channels.

better suited for work on high purity gases than the old one used by Briels et al. It is estimated that the 'pure' nitrogen of Briels et al has a contamination level of less than $0.1 \%$, while here we achieve less than $0.0001 \%$.

\subsection{Velocity measurements}

Streamer velocities have been determined on discharges in all nitrogen-oxygen mixtures and in argon. In the nitrogenoxygen mixtures, the length of a streamer is determined by the first method discussed in section 2.2 and demonstrated in figure 4. For argon, the second method must be used due to the long emission time after impact excitation. Further, it must be noted that the usable voltage range (for the C-supply) is limited to only a few kilovolts when using argon: if the voltage is too low there is no discharge at all and if the voltage is too high we get a spark that can potentially damage the camera. Therefore, we have only measured one point for argon. All results have been obtained with the C-supply. The results of the measurements at 200 mbar are given in figure 13.

The reason why we do not present measurements at 1000 mbar is that we were not able to use voltages above $45 \mathrm{kV}$ and therefore the streamers extinguish after a short propagation time, as is shown in figure 5. We also do not show measurements at 25 mbar. At this pressure, the streamers are so wide, that no fully evolved streamer channel can occur before the cathode plate is reached (see, e.g., figure 9).

The most striking feature of these measurements is the fact that all five gases show very similar velocities and voltage dependence. This is also observed at other pressures. The measured velocities have a minimum value of $(0.5-1) \times$ $10^{5} \mathrm{~m} \mathrm{~s}^{-1}$. Although the measurements at 1000 and $25 \mathrm{mbar}$ do not give a complete set of results, as for $200 \mathrm{mbar}$, we can state that at these pressures the same minimum velocity is found. We did not observe the increase in propagation velocity with increasing oxygen fraction that is reported by Yi and Williams [37]. The increase in velocity with voltage

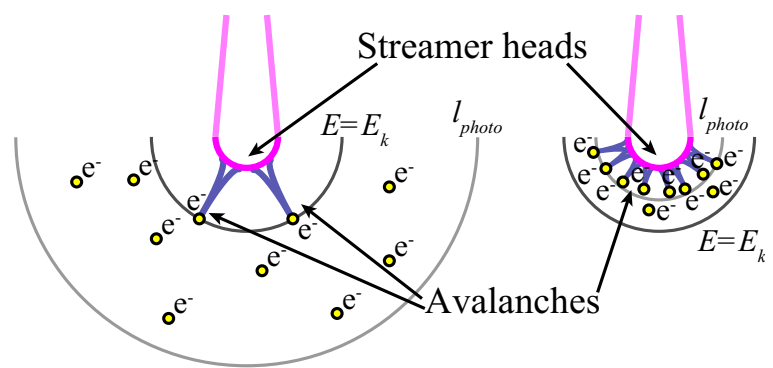

Figure 14. Sketch of the mechanism of feather formation. On the left-hand side, the photo-ionization length $\left(l_{\text {photo }}\right)$ is significantly larger than the active region where the local field exceeds the breakdown field $\left(E>E_{k}\right)$. The right-hand side illustrates the reversed situation.

is almost in quantitative accordance with the results found by Briels [45], although she published results measured at 1000 mbar in a $40 \mathrm{~mm}$ gap, while we now focus on $200 \mathrm{mbar}$ in a $160 \mathrm{~mm}$ gap.

\section{Interpretation}

The photo-ionization mechanism as described by Teich [18] is applicable to oxygen-nitrogen mixtures: the nitrogen concentration determines the number of photons in the 98 to $102.5 \mathrm{~nm}$ wavelength range; within our experiments where the oxygen concentrations range from below 1 ppm up to $20 \%$, the photon source can be considered as essentially constant (if field enhancement and radius at the streamer tip are the same).

The oxygen concentration determines the absorption length $l_{\text {photo }}$ of these photons, and therefore the range of availability of free electrons. The absorption length is about $1.3 \mathrm{~mm}$ in air at standard temperature and pressure, and it scales linearly with inverse oxygen density; the oxygen density obviously depends both on the gas density and on the $\mathrm{N}_{2}: \mathrm{O}_{2}$ mixing ratio [31].

Feather-like structures. An interpretation for the feather-like structure of the streamer channels in argon and in gas mixtures with low oxygen concentrations is the following. The small branches that form the feathers are in fact avalanches moving towards the streamer head. This is illustrated in figure 14, where two extreme cases are shown. In the first case, there is a low $\mathrm{O}_{2}$ concentration and therefore the photo-ionization length is long. When this length is much larger than the region where the electric field $(E)$ exceeds the critical breakdown field $E_{k}$, ionization is created mainly at places where the field is significantly below $E_{k}$. Therefore the created free electrons cannot form avalanches immediately after creation, or they are lost in the lateral direction where the field never exceeds $E_{k}$. Only when the streamer head comes so close that $E \geqslant E_{k}$, avalanches form. These separate avalanches are visible in the form of the feathers we see in the images of argon and pure nitrogen.

On the other hand, if there is a sufficiently high oxygen concentration in nitrogen to have a photo-ionization length that is significantly below the radius where $E=E_{k}$, all electrons that are created will immediately be accelerated towards the 
streamer head and create avalanches. Because the density of the seed electrons is much higher (the same amount of UV radiation is absorbed in a much smaller volume), these avalanches will overlap and become part of the propagating streamer head. Therefore, the streamer will appear smooth, they will easily become broad and they will not form any feather-like structures.

If photo-ionization is not present at all, but if there is some background ionization, the situation will be similar to the low photo-ionization case. Now the electrons outside the $E \geqslant E_{k}$ region will not be created by photo-ionization, but by other processes such as cosmic rays or radioactive decay (in air radon often escapes from building materials). In our stainless steel vessel filled with pure gases, the amount of radioactive radon will be orders lower than in ambient air.

Figure 14 largely resembles textbook concepts of streamer branching due to avalanches caused by single electrons; the first version of such plots stems from Raether [56]. This concept is criticized by Ebert et al in section 5.3 and figure 10 of [3]. Here it is stated that the concept that non-local photoionization leads to branching has never been substantiated by further analysis and that even if this avalanche distribution is realized, it has not been shown that it would evolve into several new streamer branches. In contrast, it is stated that the formation of a thin space charge layer is necessary for streamer branching while stochastic fluctuations are not necessary, especially because even in fully deterministic, but nonlinear models, instabilities and branching are possible. These concepts are further elaborated in [30,57-60].

Concerning the emergence of feathers rather than branches: there are stochastic avalanches, that we interpret as the 'hairs of feathers', but the streamer is apparently not in an unstable situation and does not branch. Of course, this statement is partially semantic-one also could consider the hairs as little side branches that immediately die out again. The real difference between branches and avalanches lies in the question whether the hairs do build up own space charges, or whether they just evolve in the enhanced field of the main streamer. Figure 14 suggests that the latter is the case. They die out rather after the electron avalanche has reached the main streamer and do not reach a propagating state.

If this interpretation is correct, the number of small branches could be a measure for the background ionization density when no photo-ionization exists. The branch density in the measurements at 200 mbar in pure nitrogen and argon is about $10^{2} \mathrm{~cm}^{-3}$. Unfortunately, there is very little literature regarding background ionization in pure gases in containers. Estimations for the maximum equilibrium charge density in air at atmospheric pressure are in the range of $10^{3}-10^{4} \mathrm{~cm}^{-3}$ [34]. However, this value can mainly be attributed to ionization by $\alpha$ particles emitted by decaying radon gas [34]. When we assume that the concentration of radon inside the closed metallic cylinder of our experimental set-up is some orders below the ambient value and that the pressure is 200 mbar instead of 1 bar, a background charge concentration of $10^{2} \mathrm{~cm}^{-3}$ seems reasonable.

As was stated before, the first Townsend ionization coefficient of argon is much higher than in molecular gases (at low field strengths) [54] because there are no rotational or vibrational states. Therefore the region where $E \geqslant E_{k}$ is larger. This can explain why the feather-like structures are more pronounced in argon discharges than in nitrogen discharges.

Electron attachment by oxygen. Besides photo-ionization, oxygen also plays another role since it is an attaching gas, in contrast to nitrogen or argon. This means that it removes electrons and therefore conductivity from the gas. This mechanism comes into action both ahead of the streamer head and in the streamer channel. Ahead of the streamer head and in the whole non-ionized region, it binds free electrons and makes them essentially immobile, but it can also release them again by detachment when the field exceeds a critical value. In the streamer channel, it limits the conductivity after a sufficiently long propagation time.

If, in our simple model of the feather structure (figure 14), the detachment field is higher than the critical breakdown field $E_{k}$, then the electrons at the position $E=E_{k}$ can still be attached to an oxygen molecule and the avalanches will start closer to the streamer head. However, this will only occur at very low pressures. The exact value of the detachment field depends on the vibrational state of oxygen and will therefore be different for background ionization than for photo-ionization. A detailed discussion of the role of detachment can be found in [34].

Aleksandrov et al [41] also find that the addition of $1 \%$ of oxygen to a discharge in pure argon leads to a faster decay of the streamer and requires a higher electrical energy input. However, their simulations attribute this not to electron attachment to oxygen, but to quenching of excited argon states by oxygen molecules.

A more quantitative analysis, including simulations, of the topics discussed in this section is in preparation by Wormeester, Luque, Pancheshnyi and Ebert.

\section{Conclusions}

The most simple and maybe surprising conclusion we can draw from the experiments described here is that several important streamer properties are quite similar for all nitrogenoxygen mixtures and pure nitrogen with an impurity level of less than $1 \mathrm{ppm}$. This applies to many of the overview images and zoomed images, but more so for the minimal streamer diameters and velocities. We have tested this on pure nitrogen and three different nitrogen-oxygen mixtures with nearly six orders of magnitude difference in the oxygen fraction. We do see clear differences when decreasing the oxygen concentration, but the streamers do still propagate with roughly the same velocity and their minimal diameter decreases by less than a factor of two from artificial air to pure nitrogen.

This is remarkable because it means that either the photo-ionization mechanism is not as important in streamer propagation as previously thought or that this mechanism can still provide enough free electrons, even at very low 
oxygen concentrations. When we assume that the direct photoionization mechanism is not the major source of free electrons there are a few options left for these sources:

- Other photo-ionization mechanisms than direct photoionization of oxygen by nitrogen emission are responsible for free electrons. For example, this could be step-wise ionization of nitrogen molecules, though this is unlikely at the low electron densities and high propagation velocities of the streamer tip. The measurements of Penney and Hummert [23] in pure nitrogen and pure oxygen indicate that there is photo-ionization in pure gases, although in their case, the gases are orders of magnitude less pure than our gases.

- Background ionization of the gas due to cosmic radiation, radioactivity or leftover charges from previous discharges can deliver enough free electrons for streamer propagation. The background ionization can lead to free or bound electrons. The bound electrons can be detached by the enhanced electric field of the streamer head [34]. This can be tested by modifying the background charge density in some way (e.g. adding radioactive isotopes) and studying its effect on streamer properties.

Numerical simulations of Pancheshnyi [34] show that changing the background ionization of the gas by two orders of magnitude from $10^{5} \mathrm{~cm}^{-3}$ to $10^{7} \mathrm{~cm}^{-3}$ results in only small changes (order 10-30\%) of streamer diameter, current and propagation velocity. This is in line with both the hypothesis that a very low amount of oxygen is enough to sustain photoionization and the hypothesis that background ionization is the source of the electrons needed for propagation. Note that the literature value for the background ionization in ambient air is $10^{3}-10^{4} \mathrm{~cm}^{-3}$. From the density of feathers, we estimate $10^{2} \mathrm{~cm}^{-3}$ free electrons in our 200 mbar pure gases inside our metal vessel.

We have not observed any influence of repetition frequency $(0.03-1 \mathrm{~Hz})$ on streamer morphology and propagation. Neither have we observed any tendency for streamers to follow the path of streamers from previous discharges. Therefore we conclude that the influence of previous discharges on the ionization density is either very small or does not affect streamer properties.

Yi and Williams [37] do find an oxygen-fraction dependent streamer velocity. They claim that this is caused by the difference in photo-ionization. An important difference between their measurements and ours is that they have been performed at much higher voltages $(70 \mathrm{kV}$ and above) and have a significantly different electrode geometry. Like us, they find that the streamer diameter increases with oxygen fraction. They again attribute this to photo-ionization. More photo-ionization leads to more non-local electron production and thus to a wider streamer head. Besides this, more photoionization would also reduce the required field enhancement in the streamer head, which allows the streamer head to have a blunter shape and reduces branching. Simulations by Luque et al support this [30], although these simulations treat negative streamers and we have looked at positive streamers. More detailed descriptions and simulations of these mechanisms are in preparation by Wormeester et al.
Although there are some quantitative differences (mainly a $\sim 40 \%$ reduction in measured streamer diameter), the qualitative conclusions of Briels et al [40] regarding the similarity laws have been confirmed in this work, also for the gases of much higher guaranteed purity investigated here.

\section{Acknowledgments}

SN acknowledges support by STW-project 06501, part of the Netherlands' Organization for Scientific Research NWO. The authors like to thank Guus Pemen and Zhen Liu for their help in the design of the Blumlein pulser and Sergey Pancheshnyi and Gideon Wormeester for the discussions regarding the interpretation of our experiments.

\section{References}

[1] Raizer Y P 1991 Gas Discharge Physics (Berlin: Springer)

[2] van Veldhuizen E M 2000 Electrical Discharges for Environmental Purposes: Fundamentals and Applications (New York: Nova Science Publishers)

[3] Ebert U, Montijn C, Briels T M P, Hundsdorfer W, Meulenbroek B, Rocco A and van Veldhuizen E M 2006 The multiscale nature of streamers Plasma Sources Sci. Technol. 15 S118

[4] Pasko V P 2007 Red sprite discharges in the atmosphere at high altitude: the molecular physics and the similarity with laboratory discharges Plasma Sources Sci. Technol. 16 S13

[5] Ebert U, Nijdam S, Li C, Luque A, Briels T M P and van Veldhuizen E M 2010 Recent results on streamer discharges and their relevance for sprites and lightning J. Geophys. Res. -Space Phys. at press

[6] van Heesch E J M, Winands G J J and Pemen A J M 2008 Evaluation of pulsed streamer corona experiments to determine the $\mathrm{O}^{*}$ radical yield J. Phys. D: Appl. Phys. 41234015

[7] Yan K, Van Heesch E J M, Pemen A J M, Huijbrechts P, van Gompel F M, van Leuken H and Matyas Z 2002 A high-voltage pulse generator for corona plasma generation IEEE Trans. Indust. Appl. 38 866-72

[8] Blom P P M 1997 High-power pulsed coronas PhD Thesis Eindhoven University of Technology

[9] Creyghton Y 1994 Pulsed positive corona discharges: fundamental study and application to flue gas treatment PhD Thesis Technische Universiteit Eindhoven

[10] Pancheshnyi S, Nudnova M and Starikovskii A 2005 Development of a cathode-directed streamer discharge in air at different pressures: Experiment and comparison with direct numerical simulation Phys. Rev. E (Stat., Nonlinear, Soft Matter Phys.) 71016407

[11] 2008 Cluster issue on streamers, sprites and lightning J. Phys. D: Appl. Phys. 41 234001-19

[12] 2009 AGU Chapman Conf. on Effects of Thunderstorms and Lightning in the Upper Atmosphere http://www.agu.org/ meetings/chapman/2009/bcall/

[13] Luque A, Ratushnaya V and Ebert U 2008 Positive and negative streamers in ambient air: modeling evolution and velocities J. Phys. D: Appl. Phys. 41234005

[14] Townsend J S E 1915 Electricity in Gases (Oxford: Clarendon Press)

[15] Flegler E and Raether H 1935 Untersuchung von Gasentladungsvorgängen mit der Nebelkammer Z. Tech. Phys. 11435

[16] Bradley C D and Snoddy L B 1935 Ion distribution during the initial stages of spark discharge in nonuniform fields Phys. Rev. 47541 
[17] Cravath A M 1935 Photoelectric effect and spark mechanism Phys. Rev. 47254 (abstract for APS december meeting)

[18] Teich T H 1967 Emission gasionisierender Strahlung aus Elektronenlawinen: I. Messanordnung und Messverfahren. Messungen in Sauerstoff Z. Phys. 199 378-94

[19] Teich T H 1967 Emission gasionisierender Strahlung aus Elektronenlawinen: II. Messungen in $\mathrm{O}_{2}-\mathrm{He}-\mathrm{Gemischen}$, Daempfen, $\mathrm{CO}_{2}$ und Luft; Datenzusammenstellung Z. Phys. $199395-410$

[20] Loeb L B 1936 The problem of the mechanism of static spark discharge Rev. Mod. Phys. 8267

[21] Raether H 1938 Über eine gasionisierende strahlung einer funkenentladung Z. Phys. 110611

[22] Przybylski A 1958 Untersuchung über die 'gasionisierende' Strahlung einer Entladung Z. Phys. 151264

[23] Penney G W and Hummert G T 1970 Photoionization measurements in air, oxygen, and nitrogen J. Appl. Phys. 41572

[24] Aints M, Haljaste A, Plank T and Roots L 2008 Absorption of Photo-Ionizing radiation of corona discharges in air Plasma Process. Polym. 5 672-80

[25] Zhelezniak M B, Mnatsakanian A K H and Sizykh S V 1982 Photoionization of nitrogen and oxygen mixtures by radiation from a gas discharge High Temp. 20357

[26] Morrow R and Lowke J J 1997 Streamer propagation in air J. Phys. D: Appl. Phys. 30614

[27] Kulikovsky A A 2000 The role of photoionization in positive streamer dynamics J. Phys. D: Appl. Phys. 33 1514-24

[28] Naidis G V 2006 On photoionization produced by discharges in air Plasma Sources Sci. Technol. 15 253-5

[29] Bourdon A, Pasko V P, Liu N Y, Celestin S, Segur P and Marode E 2007 Efficient models for photoionization produced by non-thermal gas discharges in air based on radiative transfer and the helmholtz equations Plasma Sources Sci. Technol. 16656

[30] Luque A, Ebert U, Montijn C and Hundsdorfer W 2007 Photoionization in negative streamers: fast computations and two propagation modes Appl. Phys. Lett. 90081501

[31] Luque A, Ebert U and Hundsdorfer W 2008 Interaction of streamer discharges in air and other oxygen-nitrogen mixtures Phys. Rev. Lett. 101075005

[32] Nudnova M M and Starikovskii A Y 2008 Streamer head structure: role of ionization and photoionization J. Phys. D: Appl. Phys. 41234003

[33] Dhali S K and Williams P F 1987 Two-dimensional studies of streamers in gases J. Appl. Phys. 62 4696-707

[34] Pancheshnyi S 2005 Role of electronegative gas admixtures in streamer start, propagation and branching phenomena Plasma Sources Sci. Technol. 14645

[35] Nijdam S, Geurts C G C, van Veldhuizen E M and Ebert U 2009 Reconnection and merging of positive streamers in air J. Phys. D: Appl. Phys. 42045201

[36] Kashiwagi Y and Itoh $\mathrm{H} 2006$ Positive surface streamers by VUV J. Phys. D: Appl. Phys. 39 113-8

[37] Yi W J and Williams P F 2002 Experimental study of streamers in pure $\mathrm{N}_{2}$ and $\mathrm{N}_{2} / \mathrm{O}_{2}$ mixtures and $\mathrm{a} \approx 13 \mathrm{~cm}$ gap J. Phys. D: Appl. Phys. 35 205-18

[38] Ono R and Oda T 2003 Formation and structure of primary and secondary streamers in positive pulsed corona discharge-effect of oxygen concentration and applied voltage J. Phys. D: Appl. Phys. 36 1952-8

[39] Briels T M P, van Veldhuizen E M and Ebert U 2008 Positive streamers in ambient air and a $\mathrm{N}_{2}: \mathrm{O}_{2}$-mixture (99.8: 0.2) IEEE Trans. Plasma. Sci. 36 906-7

[40] Briels T M P, van Veldhuizen E M and Ebert U 2008 Positive streamers in air and nitrogen of varying density: experiments on similarity laws J. Phys. D: Appl. Phys. 41234008

[41] Aleksandrov N L, Bazelyan E M and Novitskii G A 2001 The effect of small $\mathrm{O}_{2}$ addition on the properties of a long positive streamer in Ar J. Phys. D: Appl. Phys. 34 1374-8

[42] van Veldhuizen E M and Rutgers W R 2002 Pulsed positive corona streamer propagation and branching $J$. Phys. D: Appl. Phys. 352169

[43] Dubrovin D, Nijdam S, van Veldhuizen E M, Ebert U, Yair Y and Price C 2010 Sprite discharges on Venus and Jupiter-like planets: a laboratory investigation J. Geophys. Res.-Space Phys. at press

[44] Briels T M P, Kos J, van Veldhuizen E M and Ebert U 2006 Circuit dependence of the diameter of pulsed positive streamers in air J. Phys. D: Appl. Phys. 395201

[45] Briels T M P, Kos J, Winands G J J, van Veldhuizen E M and Ebert U 2008 Positive and negative streamers in ambient air: measuring diameter, velocity and dissipated energy. J. Phys. D: Appl. Phys. 41234004

[46] Smith P W 2002 Transient Electronics: Pulsed Circuit Technology (Chichester: Wiley)

[47] Liu Z, Yan K, Winands G J J, Van Heesch E J M and Pemen A J M 2006 Novel multiple-switch blumlein generator Rev. Sci. Instrum. 77033502

[48] Liu Z, Yan K, Winands G J J, Pemen A J M, Van Heesch E J M and Pawelek D B 2006 Multiple-gap spark gap switch Rev. Sci. Instrum. 77073501

[49] Nijdam S, Moerman J S, Briels T M P, van Veldhuizen E M and Ebert U 2008 Stereo-photography of streamers in air Appl. Phys. Lett. 92101502

[50] Winands G J J, Liu Z, Pemen A J M, van Heesch E J M and Yan K 2008 Analysis of streamer properties in air as function of pulse and reactor parameters by iccd photography J. Phys. D: Appl. Phys. 41234001

[51] Briels T M P, van Veldhuizen E M and Ebert U 2008 Time resolved measurements of streamer inception in air IEEE Trans. Plasma. Sci. 36 908-9

[52] Blom P P M, Smit C, Lemmens R H P and van Heesch E J M 1994 Combined Optical and Electrical Measurements on Pulsed Corona Discharges Gaseous Dielectrics vol 7 ed L G Christophorou and D R James (New York: Plenum)

[53] Shcherbakov Y V and Sigmond R S 2007 Subnanosecond spectral diagnostics of streamer discharges: I. Basic experimental results J. Phys. D: Appl. Phys. 40460

[54] The Siglo Data base, CPAT and Kinema Software. http://www. siglo-kinema.com

[55] Rep'ev A G and Repin P B 2006 Dynamics of the optical emission from a high-voltage diffuse discharge in a rod-plane electrode system in atmospheric-pressure air Plasma Phys. Rep. 32 72-8

[56] Raether H 1939 Die Entwicklung der Elektronenlawine in den Funkenkanal Z. Phys. A Hadrons Nucl. 112 464-89

[57] Montijn C, Ebert U and Hundsdorfer W 2006 Numerical convergence of the branching time of negative streamers Phys. Rev. E 73065401

[58] Derks G, Ebert U and Meulenbroek B 2008 Laplacian instability of planar streamer ionization fronts-an example of pulled front analysis J. Nonlinear Sci. 18 551-90

[59] Tanveer S, Schäfer L, Brau F and Ebert U 2009 A moving boundary problem motivated by electric breakdown: I. Spectrum of linear perturbations Phys. D: Nonlinear Phenom. 238 888-901

[60] Kao C Y, Brau F, Ebert U, Schäfer L and Tanveer S 2009 A moving boundary model motivated by electric breakdown: II. Initial value problem Physica D arXiv:0908.2521 submitted 